\title{
The Effectiveness of Group Schema Therapy to Enhance the Cognitive Emotion Regulation Strategies in Women Undergoing Bariatric Surgery During COVID-19
}

\section{Zahra Sobhani}

Shiraz University of Medical Sciences

Seyed Vahid Hosseini ( $\sim$ dr.svhoseini@gmail.com )

Shiraz University of Medical Sciences

Nazanin Honarparvaran

Hajar Khazraei

Shiraz University of Medical Sciences

Masood Amini

Shiraz University of Medical Sciences

Arvin Hedayati

Shiraz University of Medical Sciences

\section{Research Article}

Keywords: Bariatric Surgery, Group Schema Therapy, Cognitive Emotion Regulation, COVID-19

Posted Date: January 31st, 2022

DOl: https://doi.org/10.21203/rs.3.rs-1125064/v1

License: (a) (1) This work is licensed under a Creative Commons Attribution 4.0 International License. Read Full License 


\section{Abstract}

Background: Emotions, attitudes, and behaviors toward eating play a very important role in obesity and according to a schema-based approach; patients with obesity learn some coping strategies since childhood to cope with the schemas. So, this study aims to evaluate the effectiveness of group schema therapy on the cognitive emotion regulation (CER) strategies in women who underwent bariatric surgery during COVID-19.

Methods: Forty women who underwent bariatric surgery were selected and were randomly divided into two groups of control and experimental. The experimental group received ten 90-min sessions of group schema therapy. Both groups completed the CER questionnaire during pre-test, post-test and follow-up stages and data were analyzed using a multivariate analysis of covariance (MANCOVA) in SPSS software (version 20).

Results: Our results indicated that the differences between two groups in variables of adaptive CER strategies $(P=0.0001, F=31.15)$ and maladaptive CER strategies $(P=0.001, F=9.42)$ were significant.

Conclusions: The findings demonstrated that group schema therapy training can lead to increases adaptive CER strategies and decrease maladaptive CER strategies in women undergoing bariatric surgery.

Trial registration: IRCT, IRCT20180523039802N2. Registered 5 August 2020, http://www.irct.com/IRCT20180523039802N2

\section{Introduction}

The International Federation for the Surgery of Obesity and Metabolic Disorders (IFSO) recommended that all elective metabolic and bariatric procedures should be postponed until the end of the new coronavirus pandemic [1, 2]. While some studies have already been performed in a time of pandemic, the delay of operation may affect patients' health in different ways [3-5].

Obesity is an epidemic disease that is a major problem in public health, it affects over 1.5 billion people globally and is directly associated with co-morbidities such as arterial hypertension, diabetes, dyslipidemia, obstructive sleep apnea syndrome, fatty liver disease, and depression [6-8]. Bariatric surgery is one of the most effective methods for the treatment of severe obesity that might lead to a longterm reduction in weight and can enhance individuals' psychological well-being and body image satisfaction $[9,10]$. After bariatric surgery, people are expected to abandon unhealthy eating habits such as emotional eating, eating at night, drinking high-calorie beverages, and returning to the pre-surgery lifestyle [11].

Emotions, attitudes, and behaviors toward eating play a very important role in obesity, negative emotions can be a powerful predictor of overeating $[12,13]$. Overweight and obese women in comparison to the healthy group, are less aware of their emotions and have difficulty regulating their emotions, and feelings 
of loneliness or embarrassment which leads to binge eating [14]. Adaptive cognitive emotion regulation (CER) strategies are significant to well-being and successful functioning. It is vital for the commencement, motivation, and organization of adaptive behaviors. However, maladaptive CER strategies can cause maladaptive behaviors that are not conducive to each person's rehabilitation [13]. Mental health and eating behaviors may improve after bariatric surgery, and these beneficial effects may have an effect on weight reduction and maintenance [15]. Nevertheless, these advantages do not appear to endure beyond the years right away after surgery [16]. In patients with minor or considerable indications of depression, CBT was associated with weight loss before and four years after bariatric surgery [17]. While studying the need for implementing CER strategies in women who underwent bariatric surgery seems necessary in the modern world of today, research on the subject and its

application is still in its infancy not only in Iran but in other countries as well. Some studies demonstrated that cooperation in group-based psychotherapy positively affects weight loss results among postoperative bariatric patients $[18,19]$. Therefore, psychotherapy will be helpful to improve CER strategies in patients with obesity.

The goal of psychological therapy for obesity is training clients to differentiate between emotional hunger and physiological hunger and don't respond to their emotional stimuli and ruminants by eating [12]. According to a schema-based approach, patients with obesity learn some reactions and coping strategies since childhood to cope with the schemas. So, they can avoid experiencing intense emotions that resulted from early maladaptive schemas, and they are more likely to suffer from negative self-esteem [20]. Maladaptive thoughts and dysfunctional beliefs about physical shape, heaviness, and ingesting issues are important variables in obesity $[20,21]$. This treatment is planned to assist each person to spoil those negative designs of thinking, feeling, and behaving, and develop more beneficial choices to substitute them [22].

After bariatric surgery, some individuals failed in attaining or keeping up ideal weight reduction and it is far said that $18 \%$ of 500 bariatric applicants failed to attain excess weight loss after bariatric surgery. One of the foremost commonplace reasons is overeating to manage emotional distress [6, 23]. Emotional eating (EE) or eating in reaction to emotional distress is related to suboptimal weight reduction and has been found to comply with bariatric operations [24], and group schema therapy improves the eating disordered symptoms [25]. Medical weight management procedures may promote postoperative consequences, but empirical evidence is controversial [26]. So far, the effects of various psychotherapies such as motivational interviewing [27], acceptance and commitment therapy [28], and cognitivebehavioral therapy (CBT) have been conducted for post-bariatric surgery patients [29-32], but no research has examined the effect of schema therapy on CER strategies in these patients. Therefore, this study aims to evaluate the effectiveness of schema therapy on the CER strategies in women who underwent bariatric surgery.

\section{Material And Methods}




\section{Participants}

This randomized clinical trial was registered in the Iranian Clinical Trial registry under number IRCT 20180523039802 N2. The target population consisted of all women whose BMI was higher than 35 (mean $\pm S D=38 / 47 \pm 23 / 54$ ) and aged 25 to 45 years old (mean $\pm S D=36.14 \pm 10.32$ ) who underwent bariatric surgery in 2020 . The inclusion criteria were having BMI greater than 35 , receiving bariatric surgery at least 12 months ago, failing to achieve excess weight loss, and ability to read and write in the Persian language $[10,17]$.

\section{Study Design and Randomization}

We assessed 94 patients at first but 37 patients not meeting the inclusion criteria and 12 of them declined to participate in the study and 5 patients excluded for other reasons (flow diagram). Based on the formula of sample size comparison of two means with an error of $5 \%$, with a power of $90 \%$ and using the results of a similar study [17], the sample size in each group was calculated at least 18 people, which was taken to increase the accuracy of 20 patients in each group. Accordingly, 40 patients who entered the study were randomly divided into two groups of 20 with block randomization (with the size of each block equal to 4). The randomization list is generated and used using random allocation software. There was no blinding in this study (Figure 1).

The research objectives were explained to all the participants. Written and signed consent form was obtained from the participants. Additionally, all the participants in the study were assured that their information would remain confidential and that researchers would only report the group results of the study. Those who did not cooperate with the examiner were eliminated from the study. The experimental group received 10 sessions of group schema therapy with 90 minutes per session and the control group was on the waiting list. Both groups both groups completed CER questionnaire during pre-test, post-test and follow-up stages. The data were analyzed using a multivariate analysis of covariance (MANCOVA) in SPSS software (version 20).

\section{Interventions}

The study intervention was a 10-session (90 min) group schema therapy which holds online with the zoom meeting program. It was based on the Young and et all. Schema therapy protocol [20]. Briefly, this psychological treatment focused on understanding the developmental origins of the schema and relating it with obesity and relating them with currently-used cognitive emotion regulation strategies, then using the advantage-disadvantage technique to decide on whether or not to keep a schema and teaching emotion regulation techniques $[18,25]$ (Table 1$)$.

Table1: Content of the group schema therapy sessions 


\section{Sessions Content of the Sessions}

$1 \quad$ Introducing and framing the problems in schema therapy format

2 Selecting the emotional inhibition and the emotional deprivation schemas and understanding fundamental beliefs

3 Exploring the developmental origins of the schemas in childhood and relating them with currently-used coping strategies

$4 \quad$ Assessing reasons behind the formation of schemas, particularly in the interactions with parents, based on the assumptions of schema therapy

5 Schema testing and analyzing them through Socratic dialogue; reframing the schemas

$6 \quad$ Implementing limited reparenting to recognize participants' needs

$7 \quad$ Exploring the origins of the schemas through experiential techniques;

$8 \quad$ Understanding the developmental origins of the schema and relating it with obesity

9 Using the advantage-disadvantage technique to decide on whether or not to keep a schema and teaching emotion regulation techniques

10

Assessing treatment outcomes and the methods for maintaining them through flashcards

\section{Measurements}

\section{Cognitive emotion regulation strategies questionnaire (CERQ)}

The cognitive emotion regulation questionnaire (CERQ) composed of 36 five-point likert scale items used to measure CER strategies. It contains nine conceptually distinct subscales: five adaptive strategy subscales (acceptance, positive refocusing, refocusing on planning, positive reappraisal, and putting into perspective), and four maladaptive strategies subscales (self-blame, rumination, catastrophizing, and blaming others). Responses to items are measured on a five-point Likert scale ranging from 1 [(almost) never] to 5 [(almost) always], with a higher subscale score indicating greater use of a specific cognitive strategy. The CERQ has shown excellent reliability and validity. The Cronbach's alpha reliability coefficient of adaptive and maladaptive and total scores of CERQ in the current study was found to be $0.91,0.87$, and 0.93 respectively. All subscales have good internal consistencies ranging from .68 to .86 . In Iran, the results of Cronbach's alpha were in the range of 0.68 to 0.82 , indicating that the 9 subscales of the questionnaire have good reliability [33]. The range of Cronbach's alpha in the present study was 0.74 to 0.79 which shows an acceptable reliability of the questionnaire.

\section{Data analysis}

The SPSS 20 software was used for data analysis in the present study. To examine the main hypothesis in the current study analyses of covariance's (MANCOVAs) by group status (as fixed independent variable) and CER strategies as dependent variables were computed in this sample. Furthermore, baseline 
measures of adaptive CER strategies and maladaptive CER strategies were considered as covariates. The significance level for hypothesis testing was $\alpha=.05$.

\section{Ethics}

The study was approved by the Ethics Committee of Shiraz University of Medical Sciences (no.IR.SUMS.REC.1398.237), and it satisfies the Declaration of Helsinki standard [34].

\section{Results}

Table 2 shows the mean and standard deviation of adaptive CER strategies and maladaptive CER strategies scores in the experimental and control groups in pre-test, post-test and follow-up stages (Table 2). The descriptive data obtained in Table 2 indicate that the mean and standard deviation of adaptive CER strategies and maladaptive CER strategies scores in the experimental and control groups in pre-test, post-test and follow-up stages are not the same So that the scores of the experimental group in the posttest and follow-up stages improved after the schema therapy sessions.

Table 2

Mean and Standard Deviation of Dependent Variables in Pre-Test, Post-Test and Follow-up Stages among Experimental and Control Groups

\begin{tabular}{|c|c|}
\hline Sessions & Content of the Sessions \\
\hline 1 & Introducing and framing the problems in schema therapy format \\
\hline 2 & $\begin{array}{l}\text { Selecting the emotional inhibition and the emotional deprivation schemas and } \\
\text { understanding fundamental beliefs }\end{array}$ \\
\hline 3 & $\begin{array}{l}\text { Exploring the developmental origins of the schemas in childhood and relating them with } \\
\text { currently-used coping strategies }\end{array}$ \\
\hline 4 & $\begin{array}{l}\text { Assessing reasons behind the formation of schemas, particularly in the interactions with } \\
\text { parents, based on the assumptions of schema therapy }\end{array}$ \\
\hline 5 & Schema testing and analyzing them through Socratic dialogue; reframing the schemas \\
\hline 6 & Implementing limited reparenting to recognize participants' needs \\
\hline 7 & Exploring the origins of the schemas through experiential techniques; \\
\hline 8 & Understanding the developmental origins of the schema and relating it with obesity \\
\hline 9 & $\begin{array}{l}\text { Using the advantage-disadvantage technique to decide on whether or not to keep a } \\
\text { schema and teaching emotion regulation techniques }\end{array}$ \\
\hline 10 & $\begin{array}{l}\text { Assessing treatment outcomes and the methods for maintaining them through } \\
\text { flashcards }\end{array}$ \\
\hline
\end{tabular}




\begin{tabular}{|llllll|}
\hline Variables & Stages & \multicolumn{2}{c|}{ Experimental Group } & \multicolumn{2}{c|}{ Control Group } \\
\cline { 3 - 6 } & & Mean & SD & Mean & SD \\
\hline \multirow{2}{*}{ adaptive CER strategies } & Pre-test & 52.31 & 7.89 & 51.68 & 8.37 \\
\cline { 2 - 6 } & Post-test & 65.42 & 8.32 & 52.24 & 9.46 \\
\cline { 2 - 6 } & Follow-up & 64.87 & 7.69 & 51.98 & 8.58 \\
\hline maladaptive CER strategies & Pre-test & 51.43 & 8.57 & 50.96 & 9.25 \\
\cline { 2 - 6 } & Post-test & 39.52 & 8.96 & 49.33 & 8.97 \\
\cline { 2 - 6 } & Follow-up & 40.65 & 9.23 & 50.76 & 8.45 \\
\hline
\end{tabular}

To run the main statistics, at first all basic normality assumptions were computed which shows a normal distribution for running of MANCOVAs in this study. Kolmogorov-Smirnov and Shapiro-Wilks tests of normality showed normal distribution for adaptive CER strategies and maladaptive CER strategies as dependent variables during pre-test, post-test and follow-up stages (Table 3). The effect of schema therapy on CER strategies are presented in MANCOVA. In the current study before performing MANCOVA, the presumptions were firstly tested such as the Box test. Box's $M$ test for the equality of covariance across experimental and control groups showed a normal distribution of CER strategies as the basic assumption of MANCOVA ( $F=0.66=1.86, p=0.72)$. Therefore, covariance analysis can be used to examine the data. Multivariate analysis of covariance was used to test the hypothesis that schema therapy influences the CER strategies after bariatric surgery. The results are presented in Table 4 and Table 5.

Table 3

Test of Normality for Dependent Variables during Pre-Test, Post-Test and Follow-up Stages

\begin{tabular}{|llllll|}
\hline \multirow{2}{*}{ Stages } & Variables & \multicolumn{2}{c}{ Kolmogorov-Smirnov test } & \multicolumn{2}{l|}{ Shapiro-Wilks test } \\
\cline { 3 - 6 } & & Statistic & $\mathbf{P}$ & Statistic & $\mathbf{p}$ \\
\hline \multirow{2}{*}{ Pretest } & adaptive CER strategies & 0.84 & 0.124 & 0.842 & 0.104 \\
\cline { 2 - 6 } & maladaptive CER strategies & 0.97 & 0.095 & 0.912 & 0.178 \\
& adaptive CER strategies & 0.068 & 0.183 & 0.945 & 0.842 \\
\cline { 2 - 6 } Follow-up & maladaptive CER strategies & 0.092 & 0.197 & 0.932 & 0.811 \\
\cline { 2 - 6 } & adaptive CER strategies & 0.082 & 0.177 & 0.914 & 0.345 \\
\cline { 2 - 6 } & maladaptive CER strategies & 0.114 & 0.159 & 0.961 & 0.468 \\
\hline
\end{tabular}


Table 4

Effect of Eta based on Pillai's trace and Wilk's lambda test for combinational variable

\begin{tabular}{|llllllll|}
\hline P-value & $\begin{array}{l}\text { Test } \\
\text { Power }\end{array}$ & Eta & $\begin{array}{l}\text { df } \\
\text { error }\end{array}$ & $\begin{array}{l}\text { df } \\
\text { Hypothesis }\end{array}$ & F & value & Variable \\
\hline Variable & & & & & & & \\
\hline 0.001 & 0.98 & 0.79 & 12 & 8 & 8.23 & 0.76 & Pillai's Trace \\
\hline 0.001 & 0.98 & 0.79 & 12 & 8 & 8.23 & 0.34 & $\begin{array}{l}\text { Wilk's } \\
\text { Lambda }\end{array}$ \\
\hline
\end{tabular}

Table 5

The level of adaptive cognitive emotion regulation (CER) strategies and maladaptive CER strategies in experimental and control groups

\begin{tabular}{|llcccccc|}
\hline Variables & $\begin{array}{l}\text { Sum of } \\
\text { squares }\end{array}$ & DF & $\begin{array}{l}\text { Mean of } \\
\text { squares }\end{array}$ & F & $\begin{array}{l}\text { P- } \\
\text { value }\end{array}$ & Eta & $\begin{array}{l}\text { Test } \\
\text { power }\end{array}$ \\
\hline $\begin{array}{l}\text { adaptive CER } \\
\text { strategies }\end{array}$ & 114.832 & 1 & 114.832 & 31.15 & 0.0001 & 0.574 & 0.97 \\
\hline $\begin{array}{l}\text { maladaptive CER } \\
\text { strategies }\end{array}$ & 25.428 & 1 & 25.428 & 9.42 & 0.001 & 0.342 & 0.78 \\
\hline
\end{tabular}

Table 4 indicated that there is a significant difference between the experimental group and the control group in terms of the dependent variables $(p<0.001)$ and the explanatory power of 0.79 . Thus, the hypothesis of the current study is confirmed. Accordingly, it can be stated that there is a significant difference between the two groups in at least one of the dependent variables (adaptive and maladaptive cognitive emotion regulation strategies). To discover this difference, MANCOVA was used (Table 5). Moreover, the effect size coefficient indicated that the 0.79 difference between these two groups is the result of schema therapy intervention.

According to Table 5, the differences between the experimental and control groups in variables of adaptive CER strategies $(p=0.0001, F=31.15)$ and maladaptive CER strategies $(P=0.001, F=9.42)$ are significant. In this regard, the difference between these two groups was statistically significant, which means that schema therapy increases adaptive CER strategies as well as decreases maladaptive CER strategies.

\section{Discussion}

Based on the current study findings, schema therapy was effective in improving the CER strategies in women undergoing bariatric surgery during COVID-19. One possible explanation could be that there is a relationship between psychological factors on weight status, especially cognition, emotional selfregulation, psychological well-being and body image satisfaction $[9,10,12]$. Till now the effectiveness of 
schema therapy on improving CER strategies has not been studied, but performing schema therapy on the severity of eating behaviors of obese people shows that the severity of eating behaviors is directly related to early maladaptive schemas [35].

Early maladaptive schemas (EMSs) are core characteristic that taken into consideration to have progressed early in childhood through the interaction between the child temperament and the early experiences of deprivation and/or frustration related to eating psychopathology so that patients with obesity can avoid experiencing strong emotions ensued from early maladaptive schemas[36]. A negative perception of themselves leads to activating the abandonment schema and thus increasing food consumption as a way to avoid the negative emotions that result from it. These results are also similar to Peltzer and others who stated that behaviors such as overeating are designed to reduce negative emotions caused by maladaptive schemas [37].

Simpson et al. reported that group schema therapy highlighted the emotion regulation skills which had a significant alteration in early maladaptive schemas, a decrease in feelings of shame, and anxiety in females with obesity [25]. In the same vein, Chesler proposed that untreated EE is a risk factor for poor postoperative weight loss [38]. In Paul and et al. study, cognitive behavioral therapy (CBT) seems to be effective in reducing risk factors for weight regain after bariatric surgery, such as disordered eating behavior and depression [29]. The findings of Hosseini et all's study demonstrated that cognitivebehavioral therapy can lead to reduce symptoms of obsessive-compulsive disorder (OCD) in patients undergoing bariatric surgery during COVID-19 [32]. Based on the Beaulac and Sandre's findings, a shortterm CBT psychotherapy group had significant and meaningful advantages in the psychological wellbeing for post-bariatric surgery patients [30]. In the same line, Rudolph \& Hilbert reported the effectiveness of CBT for patients with behavior eating disorder after bariatric surgery [32]. David and et al. also suggested that motivational interviewing is a satisfactory and practical intervention that can improve post-surgical bariatric patients' confidence to change their eating behaviors [27]. Cotter pointed out that acceptance and commitment therapy, as well as group therapy, is satisfactory for most of the patients who experienced the bariatric medical procedure, which can provide the optimal methods to implement in the future randomized controlled trials to investigate its effectiveness [28]. Based on the findings of the present study psychological interventions can enhance the results of bariatric surgery. Thus, the results of the present study also support their findings. Concerning explaining the mentioned results and considering that most medical interventions to reduce weight have so far ignored the role of psychological factors; it is recommended that group schema therapy, which is convenient and effective on the CER strategies in women undergoing bariatric surgery, be implemented for all patients who underwent bariatric surgery.

However, definitive comments, in this regard, require more studies. Finally, it can be admitted that like any other studies, the current research contains potential limitations that may stand in way of the generalizability of its findings. For instance, the participants in this study were only females. So, it is recommended that future studies include both males and females. 


\section{Conclusion}

The findings demonstrated that group schema therapy training can lead to improve the CER strategies in women undergoing bariatric surgery. Maladaptive CER strategies can cause uncontrolled negative emotions that lead to overeating. Thus, weight management programs for patients undergoing bariatric surgery should focus on improving their control of situations related to negative emotions by improving the CER strategies.

\section{Declarations}

\section{Acknowledgments}

This article is the result of the research project with the code number 18570 at Shiraz University of Medical Sciences. Hereby, we thank and appreciate the research deputy of Shiraz University of Medical Sciences for the financial support of the project.

\section{Authors' contributions}

ZS and $\mathrm{NH}$ performed the intervention. SVH and MA did the surgery and collected data. HK and $\mathrm{AH}$ analyzed the data. ZS and HK performed a literature search and wrote the manuscript. All authors read and approved the final manuscript.

\section{Funding}

Not applicable.

\section{Availability of data and materials}

All data generated or analyzed during this study are included in this published article.

\section{Ethics approval and consent to participate}

Ethical approval was obtained from the University of Shiraz Medical Sciences (no.

IR.SUMS.REC.1398.237). Informed consent was obtained from patients.

\section{Consent for publication}

Not applicable.

\section{Competing interests}

The authors declare that they have no competing interests.

\section{Author details}


1. Colorectal research center, Shiraz University of Medical Sciences, Shiraz, Iran

2. Counseling Department, Psychology \& Education Sciences Faculty, Marvdasht Branch, Islamic Azad University, Marvdasht, Iran

3. Laparoscopy research center, Shiraz University of Medical Sciences, Shiraz, Iran

4. Research center for psychiatry and behavioral sciences, Department of psychiatry, Shiraz University of Medical Sciences, Shiraz, Iran

\section{References}

1. Raphael B. Overview of the development of psychological support in emergencies. Advances in Disaster Mental Health and Psychological Support. 2006:6.

2. Yang W, Wang C, Shikora S, Kow L. Recommendations for metabolic and bariatric surgery during the COVID-19 pandemic from IFSO.

3. Liu Z, Zhang Y, Wang X, Zhang D, Diao D, Chandramohan K, Booth CM. Recommendations for surgery during the novel coronavirus (COVID-19) epidemic. Indian Journal of Surgery. 2020 Apr;82(2):124-8.

4. Correia MI, Ramos RF, Bahten LC. The surgeons and the COVID-19 pandemic. Revista do Colégio Brasileiro de Cirurgiões. 2020 Mar 30;47.

5. Diaz A, Sarac BA, Schoenbrunner AR, Janis JE, Pawlik TM. Elective surgery in the time of COVID-19. The American Journal of Surgery. 2020 Jun 1;219(6):900-2.

6. Mingrone, G., et al., Bariatric-metabolic surgery versus conventional medical treatment in obese patients with type 2 diabetes: 5 Year follow-up of an open-label, single-centre, randomised controlled trial. Lancet (London, England), 2015. 386: p. 964-73.

7. Faria, G., J. Santos, and D. Simonson, Quality of life after gastric sleeve and gastric bypass for morbid obesity. Porto Biomedical Journal, 2017. 2.

8. Guh, D.P., et al., The incidence of co-morbidities related to obesity and overweight: A systematic review and meta-analysis. BMC Public Health, 2009. 9(1): p. 88.

9. Sjöström, L., Review of the key results from the Swedish Obese Subjects (SOS) trial-a prospective controlled intervention study of bariatric surgery. Journal of internal medicine, 2013. 273(3): p. 219234.

10. Yazdani, N.P.C., et al., Relationship between Body Image and Psychological Well-being in Patients with Morbid Obesity. International journal of community based nursing and midwifery, 2018. 6(2): p. $175-184$.

11. Schroeder, R., T.D. Harrison, and S.L. McGraw, Treatment of adult obesity with bariatric surgery. American family physician, 2016. 93(1): p. 31-37. 
12. Ozier, A.D., et al., Overweight and obesity are associated with emotion-and stress-related eating as measured by the eating and appraisal due to emotions and stress questionnaire. Journal of the American Dietetic Association, 2008. 108(1): p. 49-56.

13. Garnefski, N., et al., Cognitive coping strategies and symptoms of depression and anxiety: a comparison between adolescents and adults. Journal of Adolescence, 2002. 25(6): p. 603-611.

14. Leehr, E.J., et al., Emotion regulation model in binge eating disorder and obesity-a systematic review. Neuroscience \& Biobehavioral Reviews, 2015. 49: p. 125-134.

15. Abilés, V., et al., Psychological characteristics of morbidly obese candidates for bariatric surgery. Obesity surgery, 2010. 20(2): p. 161-167.

16. Dawes, A.J., et al., Mental health conditions among patients seeking and undergoing bariatric surgery: a meta-analysis. Jama, 2016. 315(2): p. 150-163.

17. Hjelmesæth, J., et al., Effects of cognitive behavioral therapy on eating behaviors, affective symptoms, and weight loss after bariatric surgery: a randomized clinical trial. Obesity surgery, 2019. 29(1): p. 61-69.

18. Beck, N.N., et al., Do postoperative psychotherapeutic interventions and support groups influence weight loss following bariatric surgery? A systematic review and meta-analysis of randomized and nonrandomized trials. Obesity surgery, 2012. 22(11): p. 1790-1797.

19. Saunders, R., Post-surgery group therapy for gastric bypass patients. Obesity surgery, 2004. 14(8): p. 1128-1131.

20. Young, J.E., J.S. Klosko, and M.E. Weishaar, Schema therapy: A practitioner's guide. 2006: guilford press.

21. Cooper, Z. and C.G. Fairburn, A new cognitive behavioural approach to the treatment of obesity. Behaviour research and therapy, 2001. 39(5): p. 499-511.

22. Bamelis, L.L., et al., Results of a multicenter randomized controlled trial of the clinical effectiveness of schema therapy for personality disorders. American Journal of Psychiatry, 2014. 171(3): p. 305322.

23. Zimmerman, M., et al., Presurgical psychiatric evaluations of candidates for bariatric surgery, part 1: reliability and reasons for and frequency of exclusion. The Journal of clinical psychiatry, 2007.

24. Fischer, S., et al., Emotional eating in a morbidly obese bariatric surgery-seeking population. Obesity Surgery, 2007. 17(6): p. 778-784.

25. Tewksbury, C., et al., Preoperative medical weight management in bariatric surgery: a review and reconsideration. Obesity surgery, 2017. 27(1): p. 208-214.

26. David, L.A., et al., A pilot randomized controlled trial examining the feasibility, acceptability, and efficacy of Adapted Motivational Interviewing for post-operative bariatric surgery patients. Eating behaviors, 2016. 22: p. 87-92.

27. Cotter, L., et al., Feasibility of acceptance and commitment therapy for post-bariatric surgery patients: the FAB study protocol. International Journal, 2019. 6(4): p. 198. 
28. Paul, L., C. van der Heiden, and H.W. Hoek, Cognitive behavioral therapy and predictors of weight loss in bariatric surgery patients. Current opinion in psychiatry, 2017. 30(6): p. 474-479.

29. Beaulac, J. and D. Sandre, Impact of a CBT psychotherapy group on post-operative bariatric patients. SpringerPlus, 2015. 4(1): p. 764.

30. Hosseini SV, Sobhani Z, Maytham H Al-Ganbar. Management of Obsessive-Compulsive Disorder before Bariatric Surgery with Cognitive-Behavioral Therapy during COVID-19. Obesity Surgery. 2021 Sep;31(9):4213-4215.doi: 10.1007/s11695-021-05516-5.

31. Rudolph, A. and A. Hilbert, Cognitive-Behavioral Therapy for Postbariatric Surgery Patients With Mental Disorders: A Pilot Study. Frontiers in Psychiatry, 2020. 11: p. 14.

32. Hasani, J., The reliability and validity of the short form of the cognitive emotion regulation questionnaire. 2011.

33. Aresté, N. and M. Salgueira, World Medical Association Declaration of Helsinki: ethical principles for medical research involving human subjects. JAMA, 2013. 310(20): p. 2191-2194.

34. Anderson, K., E. Rieger, and I. Caterson, A comparison of maladaptive schemata in treatment-seeking obese adults and normal-weight control subjects. Journal of psychosomatic research, 2006. 60(3): p. 245-252.

35. Pugh, M., A narrative review of schemas and schema therapy outcomes in the eating disorders. Clinical psychology review, 2015. 39: p. 30-41.

36. Peltzer, K. and S. Pengpid, Underestimation of weight and its associated factors in overweight and obese university students from 21 low, middle and emerging economy countries. Obesity research \& clinical practice, 2015. 9(3): p. 234-242.

37. Simpson, S.G., E. Morrow, and C. Reid, Group schema therapy for eating disorders: a pilot study. Frontiers in psychology, 2010. 1: p. 182.

38. Chesler, B.E., Emotional eating: a virtually untreated risk factor for outcome following bariatric surgery. The Scientific World Journal, 2012. 2012.

\section{Figures}




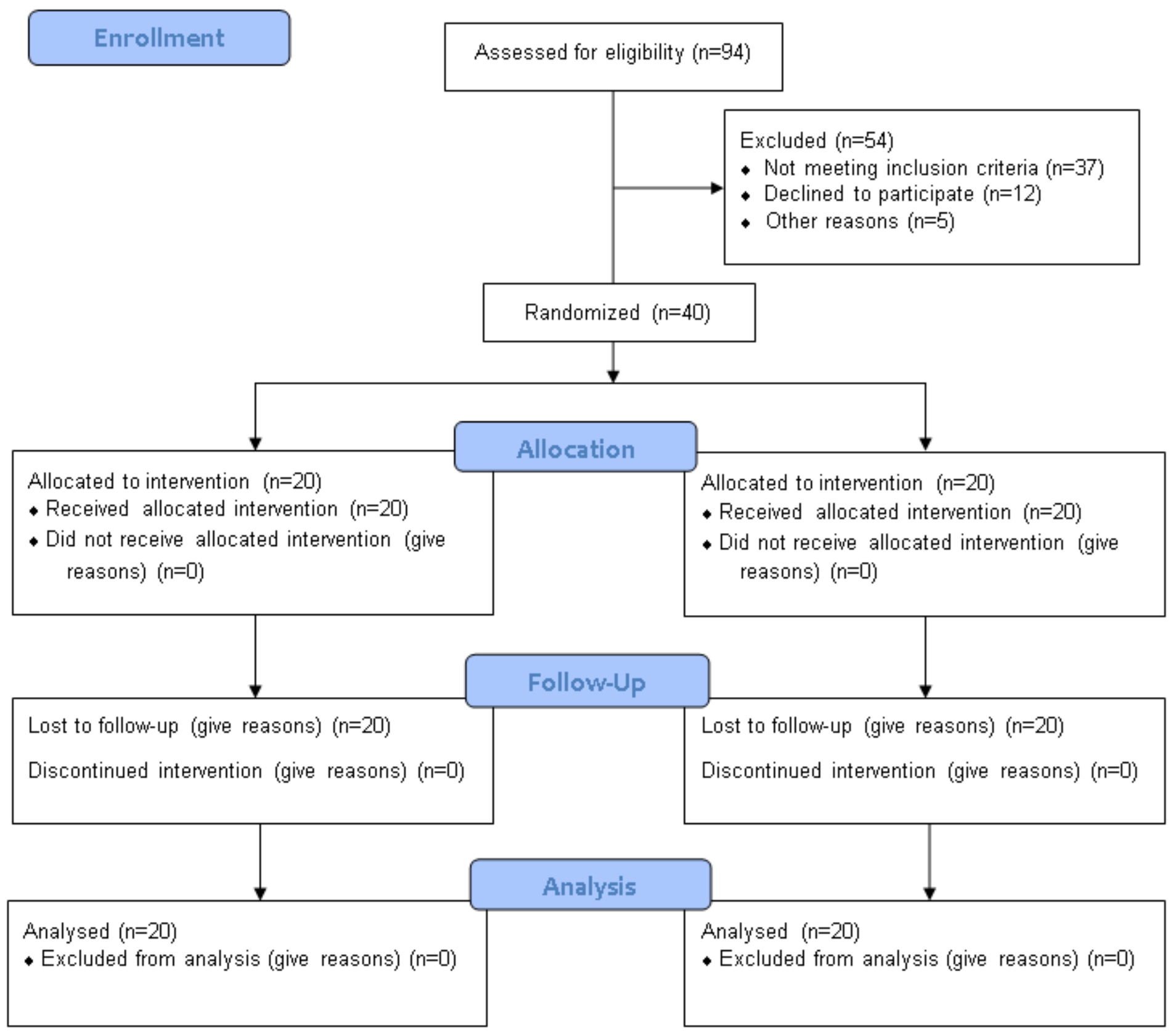

\section{Figure 1}

CONSORT 2010 flow diagram 